Interactive Semantic Analysis of English Paragraphs

- Yorick Wilks -

INTERNATIONAL CONFERENCE

ON

COMPUTATIONAL L LN"NG"UISTICS
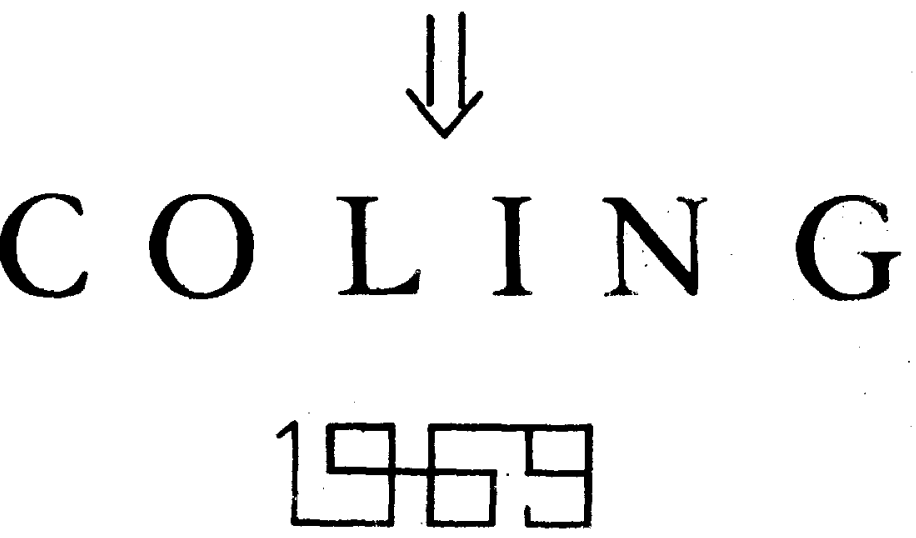

RESEARCH GROUP FOR QIIANTITATIVE LINGUISTICS

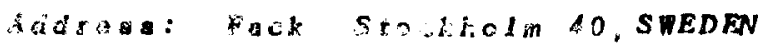




\title{
INTERACTIVE SEMANTIC ANALYSIS OF ENGLISH PARAGRAPHS
}

Abstract.

\author{
Yorick Wilks \\ 1353, N. Fuller \\ Hollywood \\ California 90046 \\ U. S. A.
}

This paper describes the use of an on-line system to do word-sense ambtguity resolution and content analysis of Fnglish text paragraphs, using a system of semantic analysis programmed in $Q 32$ LISP 1.5. The system of semantic analysis comprised dictionary codings for the text words, coded forms of permitted message, and mules producing message forms in combination on the basis of a criterion of semantic closeness. All these can be expressed within a single jystem of rules of phrase-structure form. In certain circumstances the system is able to enlarge ita own dictionary in a real-time mode on the basis of information gained from the actual texts analysed. An interpretation of the system in terms of "meaningfulness" is suggested.

Key words and phrases: semantics, language analysis, interpretation, template . CR Categories: $3.36,3.62,3.63$.

Sckmowledgements.

This work has been supported by grants from H.M. Government office of Scientific and Technical Information and A.F.O.S.R. (O.A.R.), W.shington D.C., U.S.A., administered by the Truatees of the Cambridge Ianguage Research Unit; and also by grant AFOSR F44620 - 67 - C0046 from A.F.O.S.R., monitored by Mrs. Rowena Swanson and administered by the Institute for Formal Studies, Los Angeles, California, U.S.A. The computation was done on the Q32 time-shared on-line system at systems Development Corporation, Santa Monica, California, U.S.A. 


\section{IITRODUCTION.}

In this paper I describe a syster for the on-line semantic analysis of texts of up to paragraph length. It was programmed and applied in 232 LISP 1.5 to waterial of two sorts: newspaper editorials and passages of classical philosophical argument. The immediate purpose of the analygis was to resolve the word-sense ambisuity of the texts: to tag each word of the texts to one and only one of its possible sonses or meanings, and to do so in such a way that anyone could judge the output's success or failure without knowing the coding system. The system tackles texts of up to paragraph length because I take it as a working hypothesis that many word-sense ambiguities cannot be resolved within the bounds of the conventional text sentence; there simply 1sn't enough context aveilable.

The system attempts to detect sementic forms (which I call templates) directly in coded text, and not bj means of a conventional syntax analysis. This restriction sets the present approach apart from the better-linown ones. However, an approach like the present one still has to show how to obtain the infornation contained in a conventional syntax analysis, and I shall do that below. For each paragraph of text examined the system durivos a nested structure of the semantic templatos, which can be thought of as its semantic representation. is I shall show, it may be necessary for the system to enlarge its own dictionary in an on-line mode in order to obtain such a reprasentation. From a representation, a word-sense resolution co the text is read off and printed out, since the representation contains one and only one sense representation for each constituent word of the text. 
The basic item, the template, is intended to express, in coded form, the message content of an elementary clause or sentence. Thus, if we had to analyse the sentence "The old postman is angry", I would expect to match with it a template that could be interpreted as "A certain kind of man is in a certain state". Similarly, if analysing the clause "The wicked wizard", I would expect to match with it a template that could be interpreted "a man is of a certain kind". The main hypothesis of the system of sense analysis is that one can build up a 'proper semantic sequence' of such templates as a representation of "semantically compatible" fragments of text. At the end of the paper I shall discuss the possibility of explicating the difficult notion of "meaningful language". But at the beginning I am assuming that, if a text is meaningful then its parts must cohere together in some structured way, and that "gemantic compatibility" might express that way. This working hypothesis will also mean that the word-senses that can participate in such a proper sequence will be the appropriate ones. By "appropriate senses" I mean simply the dictionary word-senses that a translator of the text would wish to distinguish from the inappropriate ones.

By way of example, I shall consider the senantic compatibilities of the fragments of a perograph to be found in a "Times" editorial in December 1966. Ls given below it has been fregmented by functions whose operations I shall describe . $\therefore$, but I shall assume that it is comprihensible as a sequence of twelve items: 
- 1 ((BRTTHINS TRANSPORT SYSTEPS ARE CHANGING)

- 2 (iND WITH IT THE TRAVIBLIING PUBLICS HLBITS)

3 (IT IS THE OLD PERMANENT WAY)

4 (UHICH ONCE MORE IS EMERGING)

L5 (AS THL PACEMGKRR)

6 (AIRLINES L LTLLY HAVE BEEN LOSIMG TRLFFIC)

7 (TO MODHRNIZED RAILWAYS)

-8 (RLILWLYS AT LAST ART BEGINNING)

(9) (TO TAKE SONE CARS

-10 (OFF THE CONGESTED SYSTEMTS TO TSTH THE WEIGHT)

11 (IF THE NEW IDE $A S$ ARE FORWLAR PRESSED)

$\mathrm{L}_{12}$ (COMML THE OF. COMMUTIR MOVEMIENT LND DORMITORY

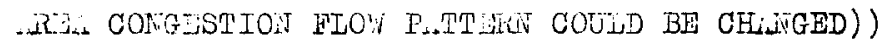

Fig. 1. A paragraph in fragment form and it's semantic compatibilities.

Iet's now look at possible semantic compatibilities between fragments of the paragraph (marked with braces in the left hand margin of the figure above).

Fragments 1 \& 2 are sementicelly compatible (both essentially assert that a structure is of a cortain sort: (1) that a system is changing, (2) that a structure is the public's.) This requires that one takes "to be of a certain sort" in its usual wide lorical sense to cover such notions as change and movement:

$4 \& 5$ are semantically compatible (both essentially assert that something is moving in some way).

$7 \& 8$ are semantically compatible (both essentially assert that the railways are near to us in time in some way). 2 \& 10 are semantically compatible (both essentially assert that something is taking or removing something). 
$11 \& 12$ are semantically compatible (both essentially assert that some structure is changing or about to change).

Notice that semantic parallelisms of this sort between fragments are sufficient to resolve at least one ambiguity in each of the pairs of fragments: for example the correct sense of "habits" for fragment 2 is "structure of behaviour", rather than the less-common "articles of dress". Thus pointing out this parallelism is also selecting the appropriate sense of "habits".

\section{THE TTXTS AND SEMUANTIC DICTIONARY}

Ten paragraph length texts were chosen for analysis: five from randomly chosen Times"editorials (data texts); and five from the works of philosophers, Descartes, Leibniz, Spinoza, Hume and witgenstein. The reason for the choice of this type of material will emerge in the discussion. Each paragraph was stored as a list of sentences on a LISP file, and an alphabetical concordance for the texts was obtained with the aid of standard routines. From this the sementic dictionary was written.

The information stored for each dictionary entry word is a list of pairs, each member of which consists of a left-hand member, which is a semantic formula such as (((THIS POINT) TO) SIGN) THING), and a right-hand rember, which is a sense description of the meaning of the corresponding formula, such as (COMPASS AS INSTRUNINT POINTING NORTH). Each such pair (called a sense-pair) corresponds to one sense of the dictionary entry word. The sense description (rigit-hand member of pair) serves only to explain to the operator, in ordinary language print-out, which particular sense of the word is being operated on at any giveñ stage of the procedure. The sense 
descriptions are not used as data for computation, except for looking at their first item to get the name of the word in question.

The purpose of the formulae is to encode, and so distinguish the different sense: of natural language words: one would expect to assign a different formula to each major sense of a word that a good dictionary distinguishes. Forralae consists of left and right parentheses and elements, where an element is one of the following 53 primitive semantic classifiers, or markers;

BD BEAST CAIT CAUSE CHANGT COUNT DO DONE FEIL FOLE FOR FORCE FRON GRAIN HaVE HOW IN KIND LER IIFE LIKIE LINE MELN MAY MORE MUCH MOST ONE PAIR PART PLANT PLEASE POINT SAME SELF SENSE SIGN SPRELD STUFT THING THINK THIS TO TRUE UP USE HIANT WHEN WHERT WHOLE WILI WORLD WRAP.

These elenents constitute the major categories of the classification of word-senses. The whole class of elements is not chosen at rendom; though as with any system of semantic markers it is difficult to justify its memborship in detail on theoretical grounds (though see 4). I shall assume here only that one has to choose sone set of markers to work with, and anyone's set of markers is always open to detalled objection. The markers are the basic elements in terms of which all the others in this system (templates, formulae etc.) are defined. So they cannot themselves be further defined, except by means of table of 'scope notes: which gives the dictionary maker some indication of the marker elements. The table contains entries like:

GRIIN: (II,IV,VI) any kind of structure or pattern. (III) structural or pattern-like. 
The Roman numerals refer to the six types of bracket groups used by the dictionary maker in constructing formulae. They are, in order, didverbial Group, Adverbial Clause, Adjunctive Group, Nominal Group, Operative Group, Operative Clause. The first two, for example, can be illustrated as follows:

I. Mdverbial Group.

((TRUE IUCH) HOW)--equivalent for "enough" used as an adverb; same function as "rather nicely" in Fnglish; can end with olement How.

II. Adverbial Clause

(ViN FROM)--same function as "out of sight" in English; cannot end with any of the elements of D4 below, and hence a II type cannot be a wellformed formula (see below) by itself.

All these six types of sub-parts of formulae can themselves be interpreted (as can the formulae) so that each left-part is dependent on the corresponding right-part. This is a non-intuitive order in IISP but is an aid to reading the formulae for Inglish speakers. This is best explained by means of an example. Thus, to take a sense-pair at rindom, say (COLOURLESS( ( (( (WHLRE SPREAD) (SENST SIGN)) NOT H.LVE) KIND) (COLOURLISS iS NOT H.VIIVG THE PROPLITY OF COLOUR)))). in explanation would be; "Colourless" is a sort; a sort indicating that something does not possess some property; the property is an abstract sensuous property of a certain sort; that certain sort has to do with spatial

$\therefore$ : t is not difficult to see that that is what (in rightleft order) the formula conveys. 
Formulae are defined recursively as follows:

D.1. A formule is a binarily bracketted string of formulae and atoms.

D.2. An atom is an element, or an element immediately preceded by "NOT".

It follows from this that an elenent is not a formula. Not all formulae can be assigned to sense-peirs, but only well-formed formulae:

D.3. The head of a formula is its last atom. (and so is the opposite of the usual notion of 'head' in LISP 1.5).

D.4. 1 well-formed formula (wff) is (a) a forrala, and (b) such that its head is one of the following elements:

HOW IIND FOLK G.IN MAN PALT SIGN STUFF THING WHOLN WORLD BE CLUSE CH.NGE DO FEEL HLVE PLI.ST FAIR SENSE WiLTT USE THIS.

3. INITISI FRLGMENTLTION OF THE TEXTS.

In initicl set of functions breaks each sentence of a paragraph up into strings of words, and, in certain circumstances, reforms discontinuous sub-strings into whole strings. The output from this process is a sentence in the form of a list of "sentence fragments", each of which (if it is not a single word) is either an elementary sentence, a complex noun phrase, or a clause introduced by a marker (such as a preposition).* So for example, the first paragraph of text is returned as on p.2 above by a function which applies the set fragfentations to each of the sentences of a paragraph in turn, and returns the paragraph as a single list of such substrings, thus obliterating the original sontence boundaries.

* These markers are largely derived from Earl . (3) 
8.

It can be seen from the example paragraph above that the functions described do not simply segment sentences in a

linear wanner. They also 'take out' certain kinds of clause

from within a sentence and append them as separate sub-strings. in example of this 'tsking out' and reforming can be seen in the example paragraph reproduced above. The first two fragments read ((BRITLINS TRLANSPORT SYSTELIS LAE CH:INGING)(WND WITH IT THE TR:VEILING PUBIICS H(ABITS)).

These are produced from a sentence that oricinally read "Britaine transport system and with it the traveliing publics habits are changing". This sort of break-up leads to an apparent grammatical 'howlor', nanely a singular subject for a plural verb. But for the purposes of semantic analysis by the present system that is not a disadvantage: it is more then outweighed by having the text cut into serantically accuptable units (see Halliday (4), for the attachment of templates to them.

The fragmented paragraphs are not passed directly to the template-matching procedure, but are first processed by a set of re-ordering functions. These inspect the fragmented out put for a paragraph and seek for qualifying phrases beginning with tararker words like 'of' and 'for'. These are delimited at their other end by the chrracter 'fo', and are placed as a whole before the word they qualify / adjectives before the preceding noun and so on. Only after this rearrangement are the fragments passed on to the matching functions. The reason for the re-ordering is that when a ternplate has been matched with a frogment, the subsequent routines seel for the qualifiers of a noun or verb only to the left of it. Thus a phrase "a book of rulas" goes to the matching routines as "a of rules fo book". 
Whe purpose of the fragnent unit is to define a unit of context between the word and the sentence, as ususlly understood. I shall call "internal" those selantic routines which operate wholly within fragmenta, and "externul" those whith scan text outsidejparticular fragment in order to resolve it:: word-senses.

\section{THE SYSTEH OF SE INTIC ANALYSIS.}

Production of ginsile baro templates

The pregent system replices each frament of text by a number of strings of formulae (frares) oonstructed from the formulae for the words of the fragment. It then searches sach frame and replaces it by a number of matching templates, or meaning structures. One can display these procedures schematically as follows:

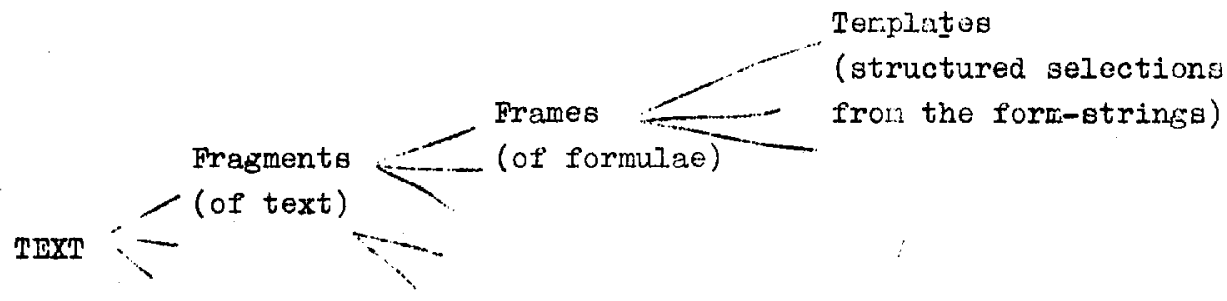

Fig. 2. nttachment of text to templates.

In the course of these procedures, therefore, each fragment of text is tagered to a number of tenplates, and so each such template is tagged to some particular selection of the wordsenses for the words of a Iragmeti. line purpose of the subsequent procedures is to redue this "fragment ambiguity" by specifying a set of striges templates, one template

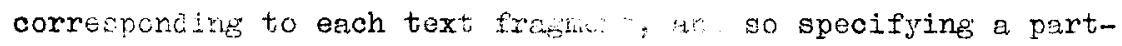
icular set of wora gensed $r$ is wore of the whole text. 
The intuitive goal is that there should be just one string of templates in the set, and hence a unique ambiguity resolution of the text. However, the possibility of a number of independent resolutions cannot be excluded a priori.

Thus the outcome of applying these procedures to a text is either nothing, or a string of sense-explanations for the words of the text. In the case where the outcome is nothing, further procedures are defined whereby the system returns, as it were, to the beginning, adjusts one or more dictionary entries in a determinate way and then tries again to resolve the text. Thus the positive outcome described may be achieved after any one of a finite number of tries. As will be seen, there is a limit to the number of possible tries; and after it has been exhausted, the system has to conclude that the text cannot be resolved by this particular method.

The procedures of resolution can be put in the form of a set of phrase-structure rules which produce a nesting of frames of formulae from an initial paragraph symbol $P$. The rules are given in their generative rather than their analytic form, but I give the "lowest-level" rulos first, because they are the ones applied at the first stage of analysis. The presentation will thus end up, rather than start, with highest level rules $P+.$. , where $P$ is a "paragraph symbol" analogous to the sentence marker, $S$, in conventional grammar.

Following what has been said above:

D.5. A frame for a fragment is a string of formulae such that each word of the fragment that has a (non-null) dictionary entry is represented by one and only one formula, and that formula has the same linear order in the as the 
corresponding word in the fragment. Thus the set of all frames consistent with this definition (and with the dictionary entries for the words of some fragment) constitutes an initial representation of a fragment in the systen.

We can now define the fundamental notion of template.

D.6. A bare template is any concatenated triple of elements that can be produced by Rules $1-6$ below. (The rules 6 . are only a sample).

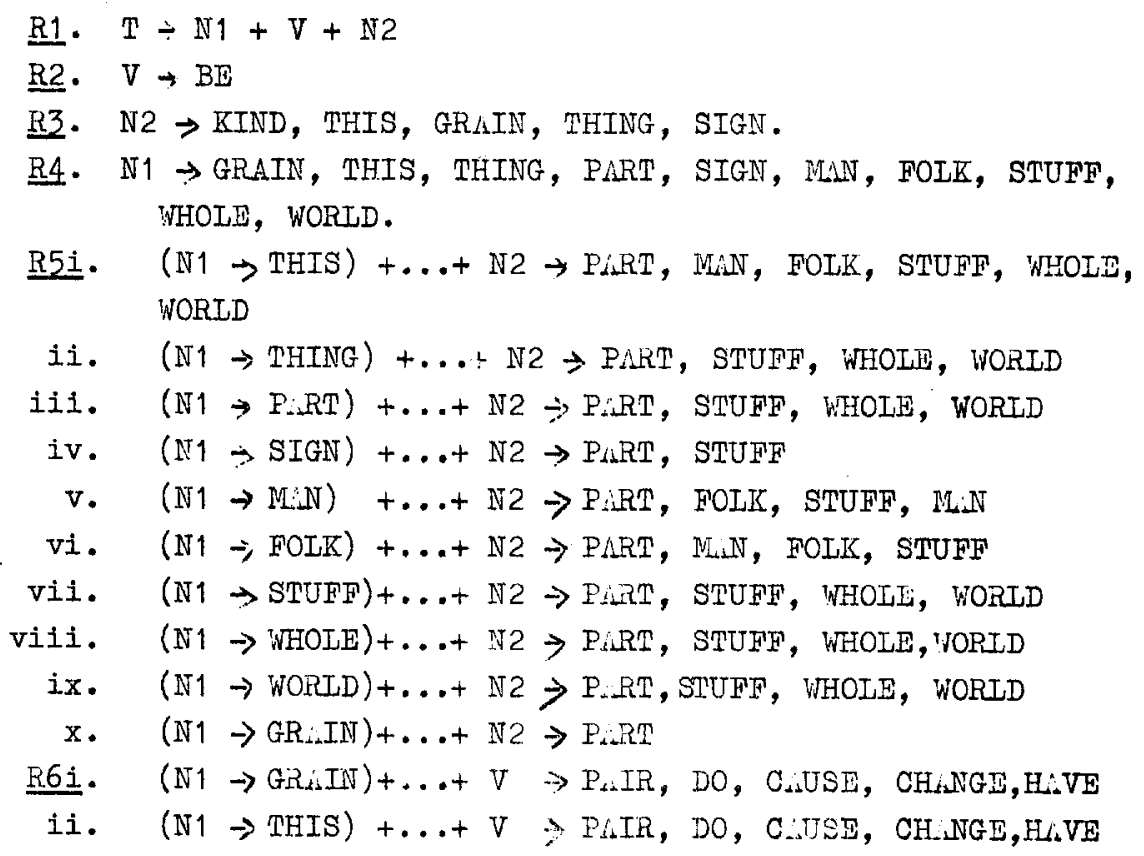

The form of rules 5 and 6 is simply a crivenicnt abbreviation of a more conventional form. For exampl. :

R5 iv. (N1 $\rightarrow$ SIGN) $+\ldots+N 2 \rightarrow P_{L A R}$ STUWF 
is simply an abbreviated expression of the two contextdependent phrase-structure mules:

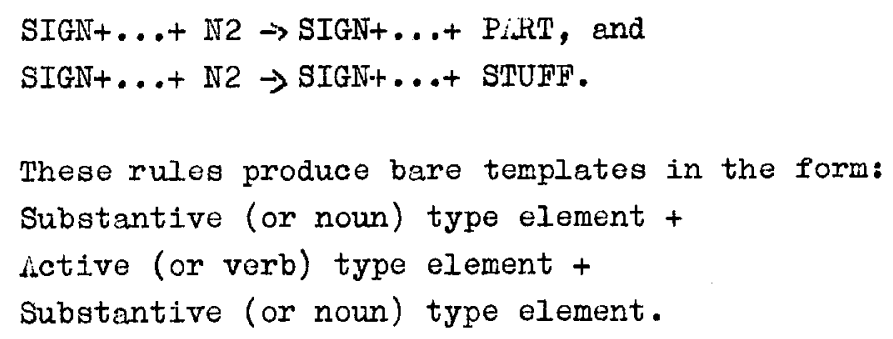

Thus MiN+H.VE+PLRT con be produced in this way, but II.NT+BE+WORID cannot. This order we call the standard order, and templates are always considered and compared in this order even if located in fragments in other (nonstandard) orders, or in "debilitated forms."

D.7 \& 8. If $\mathrm{N} 1+\mathrm{VN} 2$ represents the standard order, then $\mathrm{V}+\mathrm{N} 1+\mathrm{V} 2$ and $\mathrm{N} 1+\mathrm{N} 2+\mathrm{V}$ are nonstandard orders, and $\mathrm{N} 1+\mathrm{N} 2$ $\mathrm{NT} 1+\mathrm{V}$

N1 N1 $v \quad$ are debilitated forms.

D.9. A fragment matches with templates if a frame for it contains concatenations of heads (in left-right order) corresponding to any template produced by Rules 1-11.

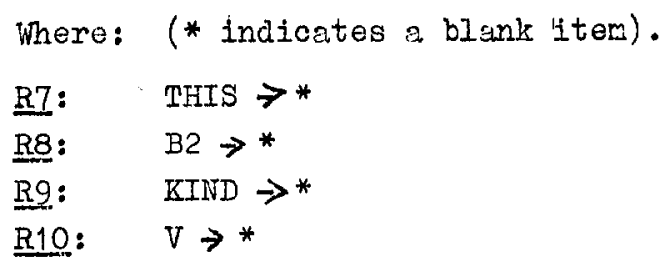




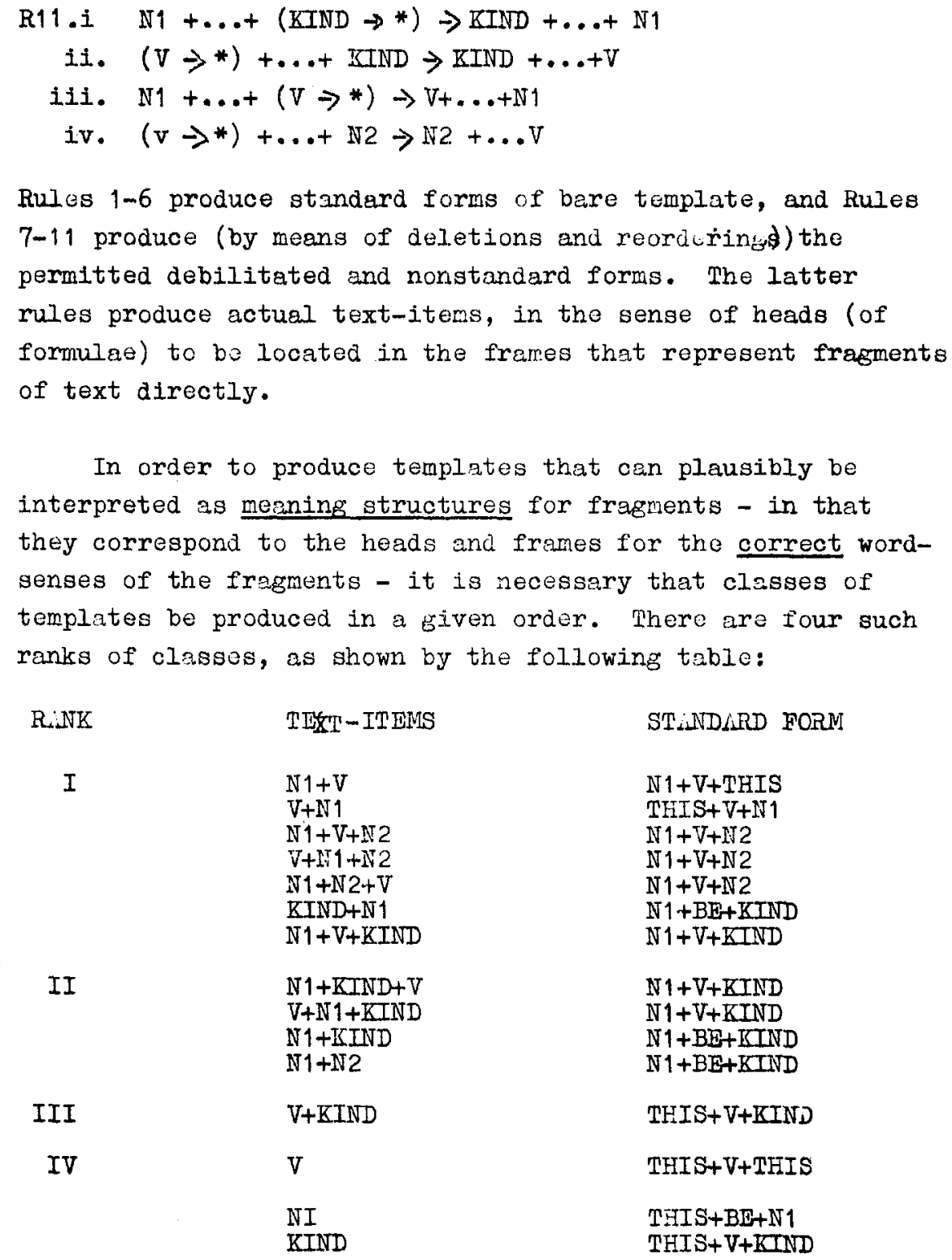

Fig 3. Preference table for bere templates. 
Since Rules 1-11 are nonrecursive, there is no problem about ordering the productions in this way. Apart from the forms fiven in the table, there are only vacuous cases such as $*+*+*$.

The above table is intended to make clear the relation between the various standard forms (in the rightmost column) and the corresponding "items in frames" produced or recognized (middle column). Thus in the senerative mode, text items are produced from the standard forms by transposition and deletion. In the analytic mode the text-items are recognized in the rank order shown, and then transposed and augmented with dummy $B S$ and THIS elements so as to be in standard form fo- further computation.

The actual function of the rank choice is best explained by example, particularly as regards the composition of Rank I, since the ranks lower than I clearly consist of "debilitated forms" and it is intuitively plausible to produce fuller forms first. This ordering is one example of the eneral rule which enables template matching to do (at least) the work of a conventional Erammar; namely, pack the frome' as tightly as possible, or, in other words, produce the fullest possible template.

The presence in rank I of the debilitated form KIND+N1 can be understood by considering, for example, the fragment:

(THE OLD TRAISPORT STSTEN).

To simplify matters I shall consider only (i) the frame consisting of representations of the appropriate senses of the words in that fragment, and (ii) the frame identical with the first except that it contains representations of OLD as substative (noun $=$ "the old people") and the active (verb) 
form of TRANSPORT. Thus, by the semantic coding system described above, those two: will contain the following heads, and in the order shown:

$$
\begin{aligned}
& \text { i. ... ...KIND) ...KIND) ...GRAIN) , and } \\
& \text { ii. ... ..FOLK) ...DO) ..GR(IN) . }
\end{aligned}
$$

Now the above rules generate both

(FOIK+DO+GRAIN) and (KIND+GRIIN)

as strings of text-items; the latter by deletion from $(N 1+B E+K I N D)$ and (KIND+N1). It is clear that if the form $\mathrm{KIID}+\mathrm{N} 1$ were not in Rank I with forms like $(\mathrm{N} 1+\mathrm{V}+\mathrm{N} 2)$ which yield (FOLI + DO+GRAIN), then a substantive phrase like this one would never receive a proper interpretation, sinco Rank I (without the form $(K I N D+N 1)$ ) would always look for an active (verb) sense for"transport" and having found one, would be satisfied.

As I have described the process so far both bare template forms (FOLIK+DO+GRAIN) and (GKAIN+BW+1rIND) would be produced. I shall show in the next section the additional procedures which produce the second of these in preference to the first Production of single full templates.

Further production rules limit the templates actually produced, and these require the notion of full template, defined as follows:

D.10. A full template is two triples of formulae such that the heads of the first triple constitute a bare template, and the second triple can be produced fror the first by the rules $12-16$.

D.11. The six formulae constituting a tull template are called text-values. 
16.

The six formulae so defined give content to the corresponding bare template (expressed by the heads of three of the formulae). The rules $12-16$ specify the other three formulae in such a way that each of them can be the qualifier of one of the formulae with a head defining part of the bare template. The rules 12-16 (not given here for reasons of space) are, in effect, rules producing an ordered pair of formulae such that the first is an appropriate qualifier for the second. Thus rule $13 i$ produces an adjective type of formula (one ending in iIIND) before a noun-type of formula, and so on.

The full templates are the items with which the system really operates. They can be illustrated by contrast with bare templates by considering fragment 3 of the paragraph excmined earlier. That fragment was "It is the old permanent way". Amons the bare templates produced for it by the system are the following two:

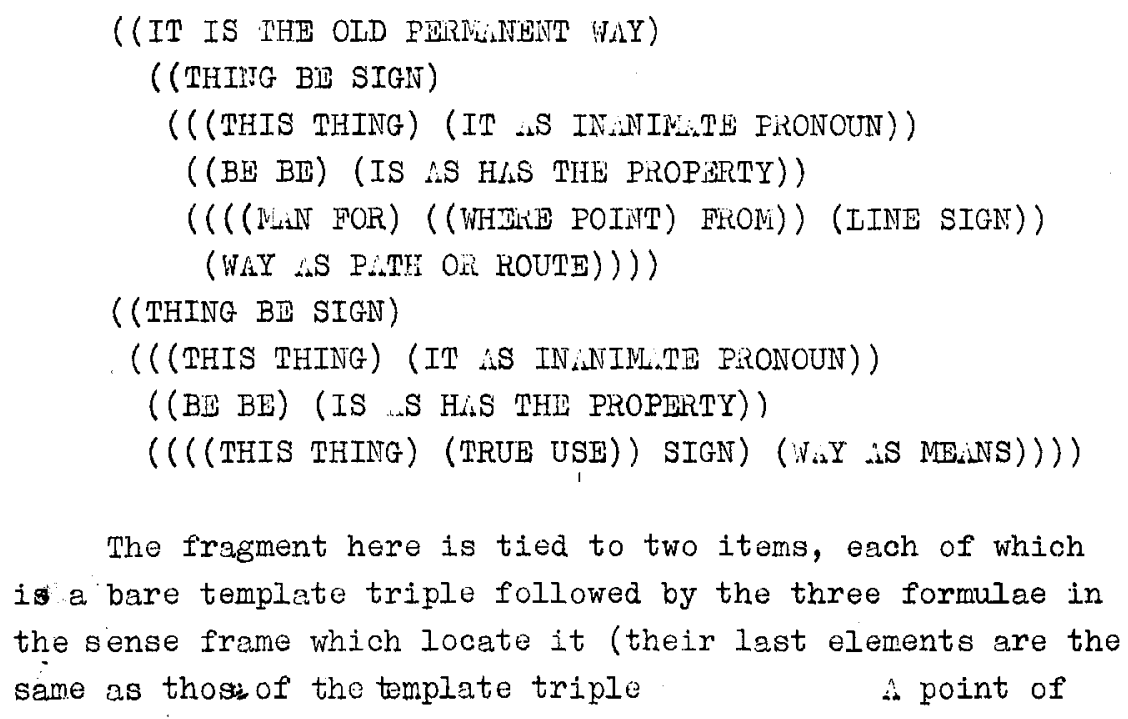


interpretation should be added here for speakers of merican English: all speakers of British Bnglish interpret "way" in this fragment as having its "path or route" sense in tris context.

The two bare templates are now expanded to full templates as follows:

((IT IS THE OLD PERNINENT WAY)

( (THING BE SIGN)

(( (ONE THING) (IT LS IN LiIIN LITE PRONOUN))

((BE BE) (IS IS H.S THE PROPERTY))

(( ((THIS THING) (TRUE UST)) SIGN) (WIY IS ME.NS))

NII NII ((NOTCH.NGE KIND) (PERMLNINT LS UNCH_NGING))))

( (THING BE SIGN)

(( (ONE THING) (IT AS IN LNINLITE PRONOUN))

((BE BE) (IS $\angle S$ HLS THE PROPARTY))

((((WHERE IN) ((WHERE POINT) FKOII)) (IINE SIGN))

(WAY AS PITH OIL ROUTE))

NII NII ((NOTCHINGS KIND) (PERMNENT IS UNCH_NGING)))))

These two items are the expansions (in franes of sense pairs) of the two bare templates. They consist of the same items as the bare template plus three formulae which are the qualifiers of the first three, (the fourth of the six is the qualifier of the first of the six and so on). In this the 'it' and 'is' have no qualifiers, honce the IISP 'III's in those positions. Bare templates other than these two were matched onto the fragment, but only these two could be expanded in this way. Hence these two were the 'survivors' and the others were rejected from furthex consideration.

When expanding in this way to produce full templates

from bare ones the lollowing metnm rule (115) is applied 
"Produce preferentially those full templates in which as many elements as possible are developed by the rules R12R14." This means producing if possible those full templates in which each element of the bare template has a formally appropriate predecessor. By means of a further rule (R16) an attempt is made to produce not only full terplates with formally appropriate internal relations, but also ones with gemantically cloge internal relations as well. That is to say, full templates such that the triple of qualifying formulae are serantically close to the formulae they respectively precede. Where,

D.12. Two formulae are said to be semantically close if:

i) they share a common pair of elerdents; or

ii) they have one or more of the following elements in common: ONE, COUNT, WORLD, WHOLE, IINE, IIND, NUST, SHLF, SPRELD, TRUE, WR.S, WHEN, WHERE, THINK; or

iii) Their cores are such that they are identical, or either is a nember of the other in the sense of a list-nember, or the left or right hand member of either core is a member of the other.

\section{Rules producing more than one template}

I can now consider the production of concatenations of the full templates described so far.

D.13. A paragraph string is any string of templates produced by the rules 17 \& 18 from the paragraph symbol $P$.

R17. $\quad P \rightarrow \mathbb{T}_{r}+T_{S}$ if $\mathrm{T}_{\mathrm{r}}$ is a full template written as a string of six formulne thus, 


$$
\left.F_{r 1}^{1}+F_{r 1}+F_{r 2}^{1}+F_{r 2}+F_{r 3}^{1}+F_{r 3}\right\rfloor
$$

where $F_{r 1}$ is a noun type; $F_{r 1}^{1}$ its qualifier (adjective type); $F_{r 2}$ is a verb type, $F_{r 2}^{1}$ its qualifier (adverb type), and so on, then

R18.

$$
\begin{aligned}
& \left(F_{s} \rightarrow F_{s 1}^{1}+F_{s 1}+F_{s 2}^{1}+F_{s 2}+F_{s 3}^{1}+F_{s 3}\right) T_{s} \\
\rightarrow & \left(F_{t 1}^{1}+F_{t 1}+F_{t 2}^{1}+F_{t 2}+F_{t 3}^{1}+F_{t 3}\right)+\ldots+ \\
& \left(F_{u 1}^{1}+F_{u 1}+F_{u 2}^{1}+F_{u 2}+F_{u 3}^{1}+F_{u 3}\right),
\end{aligned}
$$

where the values of the two template forms produced are semantically close.

D.14. Two full templates $T_{r} \quad T_{S}$ are semantically close if (with the above notation for full templates) at least two of the following pairs of formulae are (i) such that the head of the second is identical with, or in the negation"class of, the first:

$$
\left.\left(F_{r 1} F_{S 1}\right),\left(F_{r 1} F_{s 3}\right),\left(F_{r 2} F_{s 2}\right),\left(F_{r 3} F_{S 1}\right), F_{r 3} F_{s 3}\right) \text {; and }
$$

(ii) either they, or their qualifier formulae, are senantically close. These ten possible directions of connection between two full templates can be show schenatically as follows:

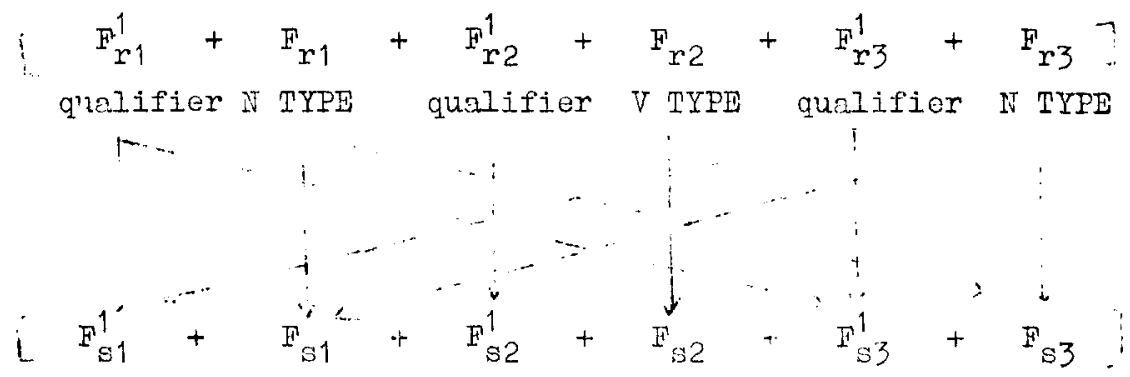

Fig 4. Connecting pattern betwe a full templates.

See note on page 31. 
Rule 18 does not, as might appear at first sight, involve self-contradiction. The sharthand form of rule writing is now beinf extended to mean that when $\mathbb{T}_{S}$ has been rewritten as $F_{S 1}+\ldots \ldots .+F_{S 3}$, then the latter nay be rewritten as the right-hand side of the second arrow.

This "expansion-concatenation" rule can be recursively applied to the initial productions from $F$. Thus at any stage in the process a paragraph string of full templates is produced. It any point the strine can be considered terminal and, with the aid of the dictionary of words and sense-pairs, the paragraph string of templates can be converted to a string of frames and so to a text of words. This is analagous to the introduction of the lexicon in any standard phrasestructuro eramnar. The dictionary entries themselves cen be put in phrase structure form. For example, if a word $w_{n}$ hes two sense pairs $S 1$ and $S 2$ in its dictioncry entry, then the sense-pairs thomselves can be put in the form $S 1 \rightarrow W_{n}$ and $S 2 \rightarrow W_{n}$ respectively. This form of the dictionary entries is useful in representing the self-Lodification of the system described below.

5. APPLICATION OF THE SYSTEM TO TEXIS.

Matching bare templates onto fragments.

Rules 1-6 above define the matching of bare templates onto a fragmented text, one bare template onto each text fragment. TEMPO is the main (top level) function that does this: it examines in turn all the frames of sense pairs for a Iragment, and so on for all the fragments of a paragraph. 
It takes as its argument a frame of sense-pairs, one for each word of a given frasment. THMPO scans each such combination in turm, starting with the frame containing all the main senses of the words in the fragment (the first one in the dictionary entry for each word). TIMPO searches for triplets of heads in the order of preference given in fis.3, above. For example, if it finds type I templates it doesn't look for any of types II-IV and so on. Each type of template is collected on a list which is the value of a different free IISP variable. If TEMPO finds nothing till it reaches the debilitated $\mathbb{N}+\mathbb{N}$ form, it replaces the $N+N$ by $N+B N+N$ (BE being the "dunmy verb"). Similarly $V+\mathbb{N}$ and $N+V$ are replaced by THIS $+V+N$ and $N+V+T$ HIS respectively (THIS keine the "durmy substantive"). The function of these durmy features is to supply a ceneral form of template for subsequent prccessing, even when it is not wholly present in the text. Suppose, for example, a fragrent consisted not of an assertion form, but of a noun phrase like "the black wizard", whore the heads of the appropriate codines for "black" and "wizard" would $\mathrm{b}$. KIND and ILIN respectively. Is there is no verb, a debilitated template of the $\mathrm{N}+\mathrm{N}$ form would match onto these two heads, and that would then be converted into $\mathbb{R} L+B N+F I N D$. which is the intuitively correct interpretation (WIZARD is BLACE). The dummy verb is added in the way described; and in cases like this, where the first head is the predicate KIND, the order of the two heads is reversed, so as to give the MN+BI+ KIND form. This transposition is defined by R11i.

\section{The internal rejection functions (matching full temrlates)}

Earlier I distinguished between intornal and external procedures. Internal rejections are whose procedures which cast out matchine; templates $l_{j}$ means of the 
expansion from bare to full templates. The main function which does this is PICKUP. It takes a fragment name as argument and constructs the TEMPO value for it. PICKUP makes a decision in the case of each templato whether or not to reject it from further consideration. Those that survive are then considered further by the external rejection procedures. The survivors from PICKUP represent a stage of ambiguity resolution beyond that given by TENPO. If, for example, PICKUP examines a template that has been natched onto a fragment containing the words round box, where $a$ template head had been attached to a forkule for box, then, hopefully, PIChUP keeps at least the templato in which round is codod by its "spatial property sense" and box" is coded by its "container" sense.

Inside PICIUP the function REFINE returns as its value a list of five sub-lists of full templates: its first sublist contains those form-close internally in four ways (as defined by rules 12-15), down to the last sub-list containing t*ose with no such closeness. PICKUP takes the first non-empty sub-list of REFIN⿴囗十 and of that returns as its value the full templates that are semantically close as well (if any).

The 'semantic parser'; resolvins a paragraph.

The top-level function PinSPARA takes as its argument a list of fragments, produces the PICKUP value for each (in the full template form given on $p .14$ ) and then parses these full templates using rules 17 \& 18 . A nesting of templates that satisfies these rules is an interpretation for the paragraph, and its word-sense content is read off and printed out (since a nesting of full templates is simply a selection of the possible word-sense assignrents for a text.) Full templates which cannot be parsed with those for other 
fragments are simply rejected This is the aternal rejection procedure reforred to earlier.

Functions celled FIT and JLM express rule 18: they test for semantic closeness between two full templates and, if such closeness is found, the two full templates are replaced by a single item with the forn of a full template. or - to put it in terms of the two function nomes - if the full templates FIT, they aro then JAMed. If the three main formulae in a full template are related to the three main formulae of another template by any three of the connectivities expressed in fig.4. abova, then the two templates FIT (are semantically close). The function JAM builds up a representation of the two templates based on their connectivities. FIT and JAM work with pessage-pairs, which are to a fragment what a serse pair is to a word.

D.15. A message-pair is a two-item list: one item is a list of the first three sonse-pairs of some full template, the other item is a list containing the name of some fragment with which the full template matches.

PiRSPiRh constructs the PICSUP value (full templates) for its list of fragments, and then builds up all possible frames of message-pairs for the paragraph. Wach frame of messae-pairs is now a possible meaning representation for the whole paragraph. PiRSPLiR then scans each frame in turn to see if it can find a right-left contiguous pair of messege pairs satisfying FIT. If it cen it deletes the first message-peir and replaces the wcond by a nessaze-pair consisting of (1) the JAM value of the tro 'parsed' full templates, and (2) a list of the names of he fitting fragnents. 


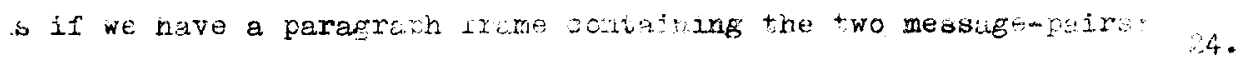

((GRITLIHS TLLANSPORT SYSTEM _ZF CH.JGING)

(( (WHOLE GR_IN) (SYSTEM _.S LN ORG_NIZLTION))

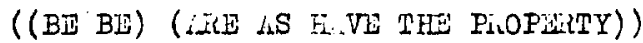

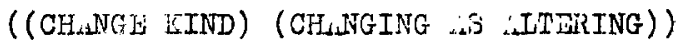

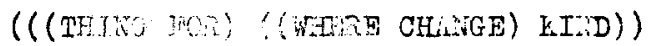

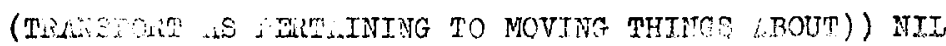

and

IIII ))

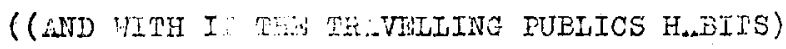

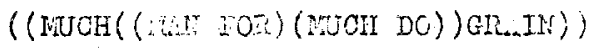

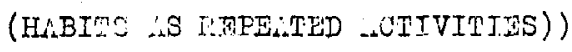

( $(B D E D$ (DUINY ) )

(( (WHOTI FCITI) RITH)

(PUPIICS .Е CUNNECTHL WITH THE WHOLY PBOETA))

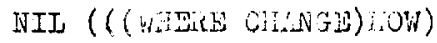

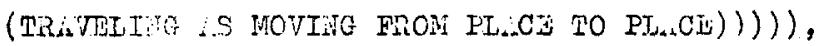

then the two full templates in those mosseft-pairs are a fittine pair, wo shall expoct then to be ruplanod in the strine by the form:

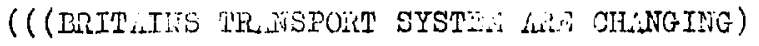

(WITIA IT IFEY TR:VELIING ITUBLICS HABITS))

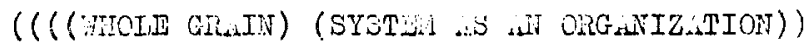

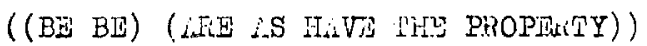

((CH.INGI KIND) (CHANGING AS IITZIIIG ) )

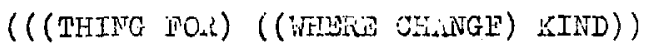

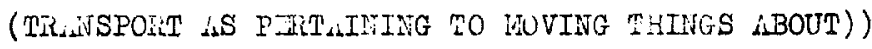

NII ( ( WHZZZE CHLNGE) WEOW)

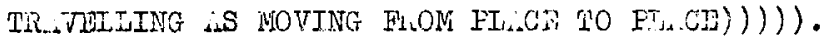

This fittine together, or persine, of message-pairs expresses the senantic conpatibility between the corresponding

fragments disoussed earlier. Pifspilus rewrites such strings 
25.

of message-pairs recursively, trying to reech a two item list which (by rule 17) is $P$ the paragraph syrabol. If this point is reached the corresponding sense-resolution is read off and printed out for the paragraph in the following form: each fragment is given with the list of sense expressions for all the words in it which are resolved (or which had only a single sense entry initially, and so are trivially resolved); a list is also given of words not resolved (if any).

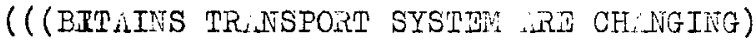
((WORDS RESOLVED IN FRLGMENT)

((TRINSPORT AS PERTAINING TO MOVING THINGS :BOUT) (BRITLILTS LS HAVING THE CHALACTERISTIC OF A PliTICUIiR PIIT OF THE WOIDD)

(SYSTEN IS IN ORGLNIZUTION)

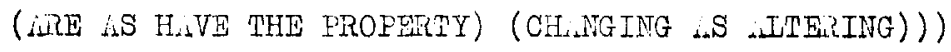

((WOIDS NOT ILSSOLVED IN FIR,GNENT) NIL))

((WITH II III: TALELIING PUBLICS HABITS)

((WORDS IRESOLVED IN FRLIGNEIT)

((TR:TIELIING IS MOVING FROM PL:_CE TO PLLCE)

(IT LS INLITIMLTE PRONOUN )

(H.BITS ${ }_{22}$ S REPEHATED ${ }_{11}$ CTIVITIES)) )

((WOLDS IYON RTSOLVED IIT FRLGRENTI) NIL)

fig. 5. First two fragments of the resolved output for $\mathrm{e}$ text paragraph.

The original English for the first two fragments of that paragraph was "Britain's transport system and with it the travelling public's habits are changing".

The sense constructur. procedure.

A procedure was built in to the systen to deal with the 
cases where the system returned (NO RESOLUTION ALI PATHS $B L O C L E D)$ at the teletype. This situation could arise for a number of reasons; the text fragments did not cohere together sufficiently; a vital word sense had been left out of the dictionary; or a word in the text was being used in a new and original sense. An obvious suggestion for tackling this is to allow the word dictionary to enlarge itself: to supply an additional sense entry for the word that is holding the procedure up, if it can be found. Such a construction could thought of as adding a new rule $F-a$, where $F$ is a formula and a word nawe, and so expanding to a new rule system as the system adjusts to the particular text.

In practice PARSPARA examined the value of a free variable BESTPARS each time it failed to parse a frame completely. It stored as the value of BESTPARS the parsing tree containing the template that had been rewritten least. It seemed a good first guess at the recalcitrant word that it was in template that 'cohered' least with its neigbours. If all the frame blocked PARSPARA would print (CONSTRUCTER MODE) and evaluate a function of no variakles called CONSTRUCTER. This function controls all subsequent operations via the READ and PRINT functions at the teletype. CONSTrUCTER lookg at the value of the recalcitrant template in BESTPAFS and suggested that a word in the corresponding fragment have its dictionary of sense pairs enlarged by 1dentifying the recalcitrant word with the nost 'semantically close' word in the paragraph. If the operator accepts the system's suggestion at the teletype, the system is rerun with the enlerged dictionary to try and get a resolution. In such a case (or if none of the system's suggestions are acceptable to the operator) the system returns to the normal operating mode. This procedure was not called upon for the newspaper paragraphs, but it produced some interesting suggestions in the case of two of the philosophical paragraphs. 
In CONSTRUCT: MODE dialogues like the following are possible:

(CONSTRUCTER MODE)

((NO RESOLUTION ALL PATHS BLOCKBD) (BEST PARSING CONTAINS)

(( (( (KIITD SIGN) (ATTRIBUTE AS A PARTICULAR KIND OF PROFERTY))

((BD BE) (DUNWIY )

((S.ING RIND) (S.ITS LS IDENTICAL))

((KHOLE (NIST (KIND SIGN)))

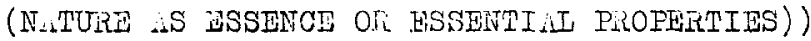

NII NII)

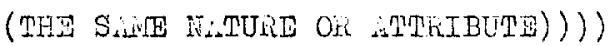

(RECALCITRANT TTMPLITE IS FOR)

(THE SAIE NATURT OR AITHEDUTE)

(CONTINUE YES ORT HO)

YES

(SUGGIST ATTKIBUTE AS NNTURE (SHALI I TRY IT YMS OR NO)) YES

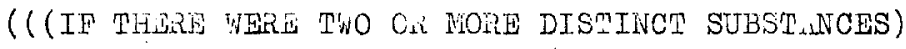

((WORDS LESOLVIAD IN FRLGMLNT)

((THERE $\therefore$ S $\Lambda \mathrm{T}$ \& POINT)

(WERI ISS EXISTED)

(OR LS DISJUNCTION)

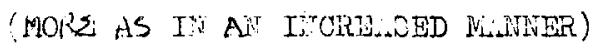

(DISTINCT AS DIFFERLITT) (SUBST.ANCES AS SORTS OF

THING)))

((WORDS NOT RTSOLVHD IN FR.:GMLITT)

(TWO .

(((COUNT SIGN) (TWO iSS is NUMBEIR))

((COUNT KIND) (TWO AS FiVING TET PROPIRTY OF TWOITY $))))$ )

fig. 6. Dialogue in CONSTRUCTER MODE together with first part of subsequent resoiution. 


\section{$\underline{6} \underline{\text { DISCUSSION }}$}

One of the main difficulties in coding for, and evaluating, a system like this one is the necessary vagueness of some of the sense-entries (especially evident in words like 'it' and 'is'). Noktrele'ss I claim that the present system could constitute a tentative criterion for meaningfulness: a text is meaningful if and only if a system like tins present one can resolve it. It is easy enough to get a necessary criterion on the ground that one needs to be able to tell in what senses the words of a text are being used in order to call it meaningful. I have arsued at length elsewhere that it is possible also to justify the corresponding sufficient one (8). The establishment of such a criterion would be of some interest in the cases of the five philosophical paragraphs, since it was texts like these that Carnes (2), and the 'Logical Syntax' school eenerally, said could be shown to be meaningless on the basis of a system of analytic rules, thoush they never in fact constructed such a system. The criterion sugfested here would only be one of degree (in terms of the number of applications of the sense-constructer procedure a text required for resolution). That is perhaps the only acceptable form that a criterion of meaningfulness could take, as there seems something absurd about an attempt to set an absolute bound to the neaningful.

Inother speculative interest of the present system might be its application to the speech patterns of schizophrenics. Schizophrenic discourse seems (6) to be meaningful within the bounderies of units of the same order of length as the clause or phrase. The troubie is that these units don't seem to fit together in a coherent way in the schizophrenic's 
speech pattern: is system of the present sort, which tries to make such items cohere, might conceivably provide a measure of "semantic disorder" in such cases.

4h number of connexions can be made also between the semantic structure assigned to a text by the present system and that assigned by formal logic. These connexions have beon investigaien in the cases of the five philosophical paragraphs, which have a form sufficiently like the one required by formal logic. These connexions are of some interest in view of the almost total neglect of the sense-ambiguity of natural lancuace words by formal logic.

One can, for example, interpret the present system so as to craate a notion of "valid and usefull argument. It has long been recognised that an argument can be formally valid (and ever have true premisses) and yet bo conpletely useless. Ihis is usually due to a genuine ambiguity in the argument. For example, the following is perfectly valid: "ill kings wear crowns, all onowns are coins, thorefore all kings wear coins". And, within the coitext of each premiss, each premiss is true. (In the "numismatic world of discourse", for example, the second is true).

in argument could be deemed "valid and useful" if it is formally valid and if the present system assigns to it a consistent and complete irterpretation. I an usins the terms 'consistenl' and 'complote' in a way similar to Bobrow's (1) use of them: ain interpretation is complete if the system assigns an interpretation to each key term in the arsument, and consisten if it assigns the same interpretation(wordsense) to every occurrence of a terin. Thus the areument above 
30.

world not pass the 'usefulness' criterion, since a proper ambiguity-reaolver would assign dj.ferent interpretations to the two cccurrences of the key term 'crown'. 
RIEFERENCES

1. Bobrow, D.G. Natural Input for a Computer Problem-Solving System. Ph.D. Thesis, M.I.T. (1965)

2. Carnap, R. The Logical Syntax of Language, iroutledge, Lond on (1937)

3. Earl, I. Silgorithm for sutomatio Clause delimitation in Dnglish sentences. Lockheed Missiles and Space Co., Tech. liept. 5.13.64. 5. (Tarch, 1964)

4. Greenbers, J.H. Universals of Language. M.I.T. Press, (ed) Caribridge Mass. (1963)

5. Halliday, M. Some aspects of the thenatic orsanization of the English Clause. RiND lienorandum 5224 (January, 1967).

6. Laing, R.D. The Divided Self. Tavistock Publications, London (1960)

7. satz, J., and in integrated theory of Iinguistic Postal, P. Descriptions. M.I.T. Press, Crmbridge, Mass., (1964).

8. Wilks. Y. wrgursent and Proof in Metaphysics, from an Finpirical Foint of View. Ph.D. Thesis, Cambriage. (1968)

tc page 19:

The negation class of elements for each element is derived jnductively by a separate procedure. The notion onvclved is like that of logical contrarysan element and any member of its nggation class are partiy syzonymous and partiy exclusive.For example, an entity can be basically a sTUis or basically a THING;it cannot be both so each of these elements is in the negation class of the other. 